\title{
Erratum to: miR-143 inhibits tumor progression by targeting FAM83F in esophageal squamous cell carcinoma
}

\author{
Yu Mao ${ }^{1,2} \cdot$ Jia Liu $^{3} \cdot$ Dakai Zhang $^{3} \cdot$ Baosheng Li ${ }^{1,2}$
}

Published online: 24 October 2016

(C) International Society of Oncology and BioMarkers (ISOBM) 2016

Erratum to: Tumor Biol. (2016) 37:9009-9022

DOI 10.1007/s13277-015-4760-9

In the original publication of the article, the bar chart for Figures $6 \mathrm{~B}, 7 \mathrm{~B}$ and $8 \mathrm{~B}$ were published erroneously.

The corrected Figures 6,7 and 8 are shown in the next page.

The online version of the original article can be found at http://dx.doi. org/10.1007/s13277-015-4760-9.

\footnotetext{
Baosheng Li

baoshli1963@163.com

1 National Clinical Research Center of Cancer, Key Laboratory of Cancer Prevention and Therapy, Tianjin Medical University Cancer Institute and Hospital, Tianjin 300060, China

2 Department of Radiation Oncology (Chest Section), Shandong's Key Laboratory of Radiation Oncology, Shandong Cancer Hospital, Shandong Academy of Medical Sciences, Jiyan Road 440, Jinan 250117, Shandong Province, China

3 School of Medicine and Life Sciences, University of Jinan-Shandong Academy of Medical Sciences, Jinan, China
} 
A

\section{KYSE410}

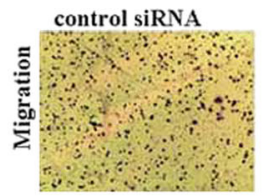

FAM83F siRNA
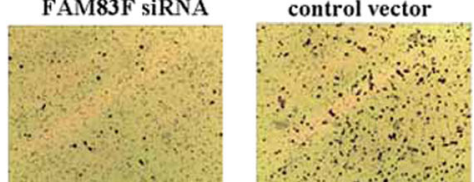

FAM83F vector
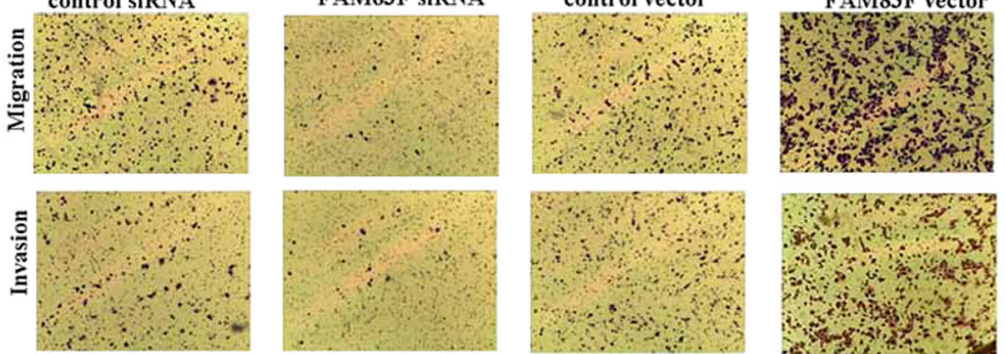

ECA109
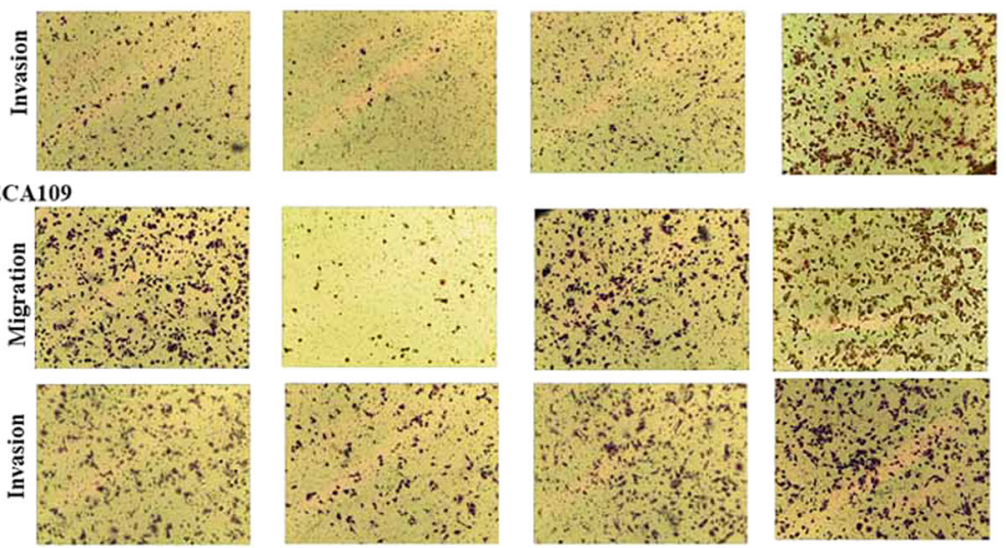

B
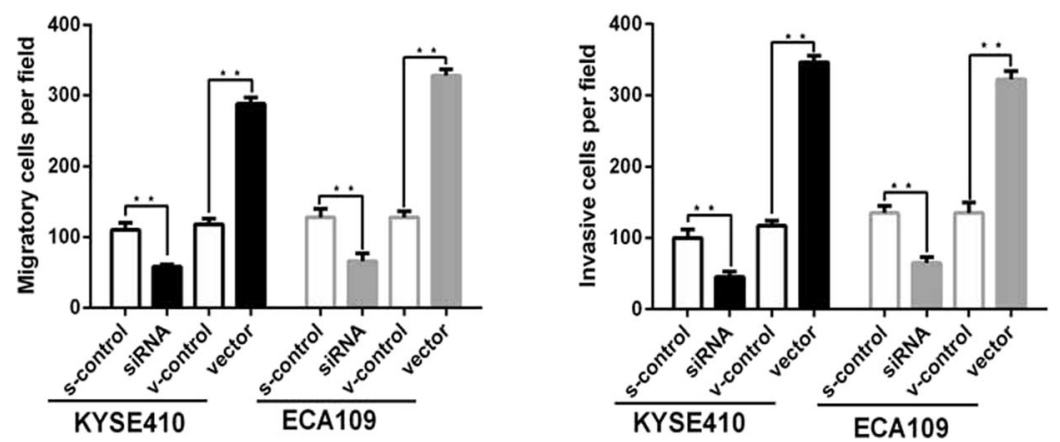

Fig. 6 The effect of FAM83F expression on migration and invasion of ESCC cells. a Representative images of migrating and invasive cells per field after transfection of ESCC cells. b Quantification of migrating and invasive cells per field after transfection of ESCC cells 
A

KYSE410
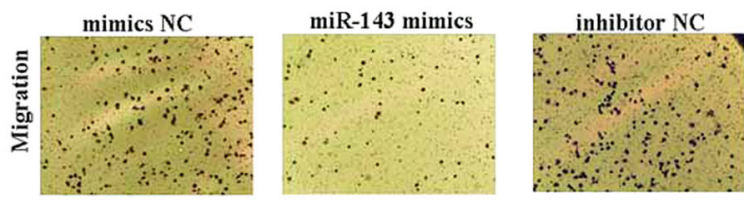

miR-143 inhibitor
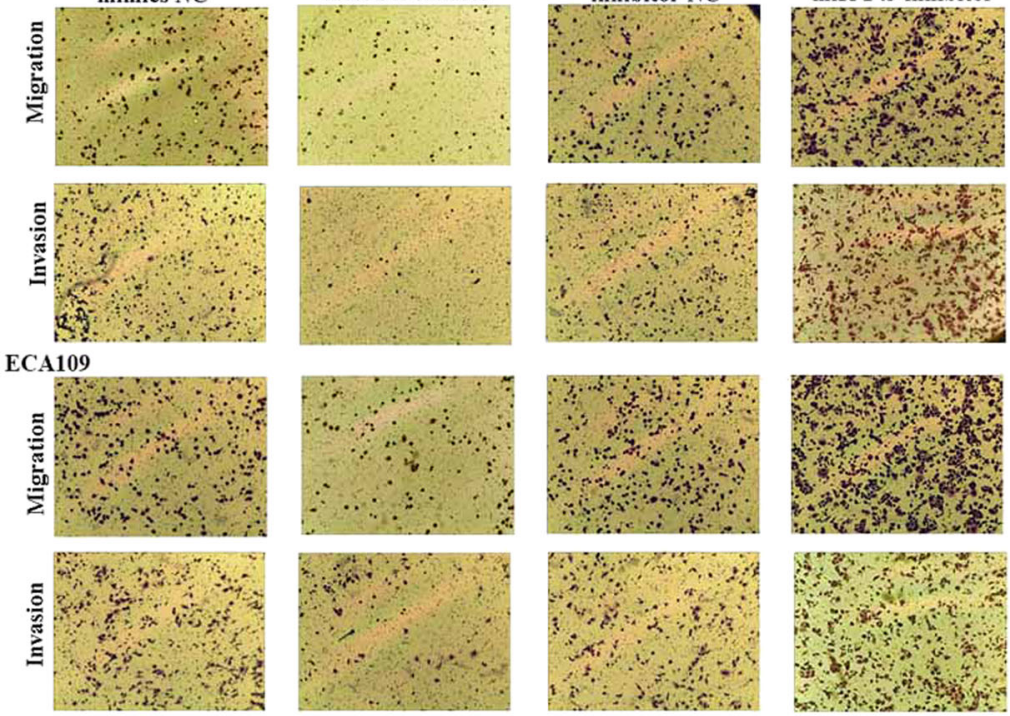

B
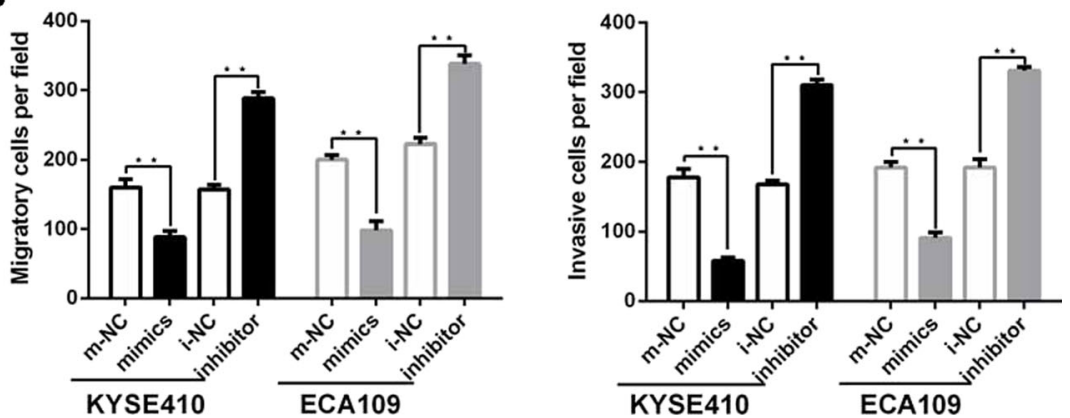

Fig. 7 A representative graph for laccase production and yeast cell growth of Yarrowia lipolytica YL4 in PPB medium during 7 days. All the results presented are the mean values \pm SD for three independent replicates 
A

KYSE410
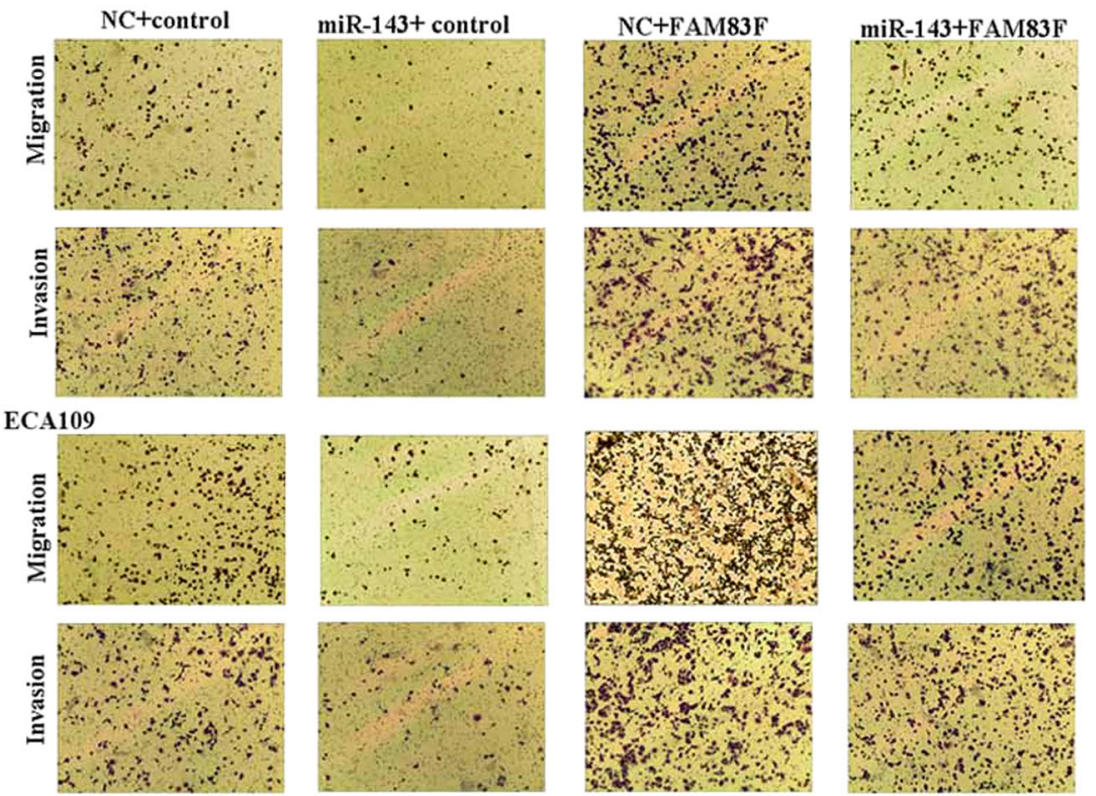

B
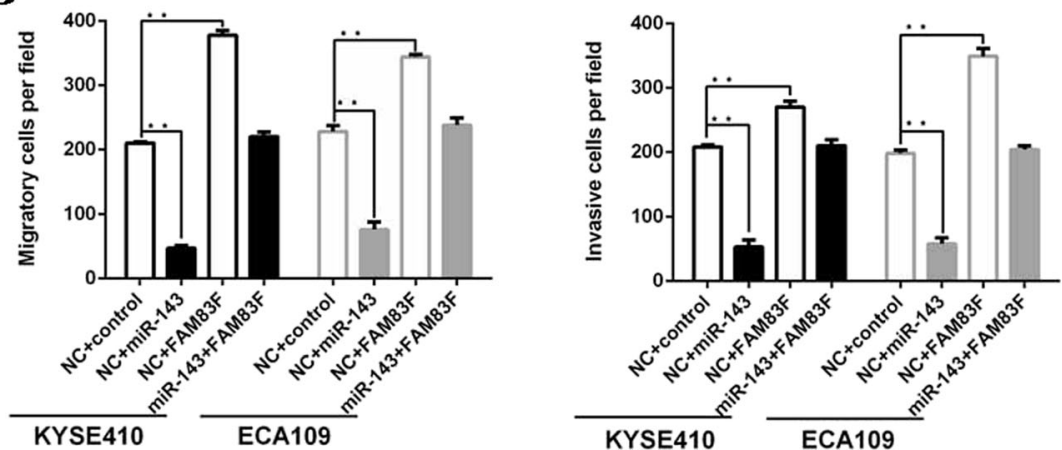

Fig. 8 miR-143 inhibits the migration and invasion of ESCC cells by targeting FAM83F. a Representative images of migrating and invasive cells per field after transfection of ESCC cells. b Quantification of migrating and invasive cells per field after transfection of ESCC 